\title{
Caracterização dos atendimentos ambulatoriais de urgência em minas gerais e implicações no papel da enfermagem
}

\author{
Characterization of ambulatory emergency care in minas gerais and implications on the role of \\ nursing
}

Caracterización de la atención de emergencia ambulatoria en minas gerais e implicaciones sobre el papel de la enfermería

Júlia Lancaster de Carvalho Freitas ORCID: https://orcid.org/0000-0001-8261-9496 Universidade do Estado de Minas Gerais, Brasil E-mail: julialancaster10@gmail.com

Marina Correa Alves dos Reis ORCID: https://orcid.org/0000-0001-7100-6790 Universidade do Estado de Minas Gerais, Brasil E-mail: marinareis9810@gmail.com

Fernanda Marcelino de Rezende e Silva ORCID: https://orcid.org/0000-0003-2236-7009 Universidade do Estado de Minas Gerais, Brasil E-mail: fernanda.silva@uemg.br

Karla Amaral Nogueira Quadros ORCID: https://orcid.org/0000-0002-3750-4873 Universidade do Estado de Minas Gerais, Brasil E-mail: kanq@bol.com.br

Eduardo Nogueira Cortez ORCID: https://orcid.org/0000-0003-4974-1451 Universidade do Estado de Minas Gerais, Brasil

E-mail: eduardonogueiracortez@gmail.com Heber Paulino Pena ORCID: https://orcid.org/0000-0002-9122-6827 Universidade do Estado de Minas Gerais, Brasil E-mail: heberppena@yahoo.com.br

Flávia de Oliveira ORCID: https://orcid.org/0000-0002-9044-6588 Universidade Federal de São João del-Rei Brasil E-mail: flaviadeoliveira@ufsj.edu.br

Regina Consolação dos Santos ORCID: https://orcid.org/0000-0002-7393-3210 Universidade do Estado de Minas Gerais, Brasil E-mail: regina.consolacao@uemg.br

Túlio Resende Freitas ORCID: https://orcid.org/0000-0002-2289-2201 Universidade Federal de Minas Gerais, Brasil E-mail: tuliorfreitas@gmail.com Silmara Nunes Andrade ORCID: https://orcid.org/0000-0002-1975-0827 Universidade do Estado de Minas Gerais, Brasil E-mail: silmara.andrade@uemg.br

\begin{abstract}
Resumo
Objetivo: Realizar a descrição os dados disponibilizados pelo Sistema de Informações Ambulatoriais sobre os atendimentos de caráter de urgência descrevendo o papel da enfermagem e sua relevância. Métodos: Trata-se de um estudo descritivo de abordagem quantitativa. Foi realizada uma análise de série temporal dos atendimentos de caráter de urgência do Estado de Minas Gerais, no período de janeiro a dezembro de 2019, através do Sistema de Informações Ambulatoriais. Resultados: A partir da computação dos dados foi possível observar variações quanto à quantidade de atendimentos de caráter de urgência, quanto aos mesmos realizados por profissionais enfermeiros e quanto aos atendimentos por nível de complexidade, com destaque para o mês de dezembro. Conclusão: É necessário valorizar os profissionais de enfermagem, fortalecer a Atenção Básica como porta de entrada do sistema de saúde e promover a educação em saúde, esclarecendo aos usuários as atribuições de cada serviço.
\end{abstract}


Palavras-chave: Enfermagem; Emergências; Assistência ambulatorial.

\begin{abstract}
Objective: It aims to carry out the description of the data provided by the Ambulatory Information System on urgent care, describing the relevance and role of nursing. Methods: This is a descriptive study with a quantitative approach. A time series analysis of emergency care in the State of Minas Gerais, from January to December 2019, was carried out through the Ambulatory Information System. Results: Through the analysis of the data, it was possible to observe variations in the amount of emergency calls, in relation to the same ones performed by professional nurses and in terms of calls by level of complexity, with emphasis on the month of December. Conclusion: It is necessary to value nursing professionals, strengthen Primary Care as a gateway to the health system and promote health education, clarifying the responsibilities of each service.
\end{abstract}

Keywords: Nursing; Emergencies; Outpatient care.

\title{
Resumen
}

Objetivo: Realizar la descripción de los datos proporcionados por el Sistema de Información Ambulatoria sobre atención urgente, describiendo la relevancia y rol de la enfermería. Métodos: Se trata de un estudio descriptivo con enfoque cuantitativo. A través del Sistema de Información Ambulatoria se realizó un análisis de series de tiempo de la atención de emergencia en el Estado de Minas Gerais, de enero a diciembre de 2019. Resultados: A través del análisis de los datos, fue posible observar variaciones en la cantidad de llamadas de emergencia, en relación a las mismas realizadas por enfermeras profesionales y en términos de llamadas por nivel de complejidad, con énfasis en el mes de diciembre. Conclusión: Es necesario valorar a los profesionales de enfermería, fortalecer la Atención Primaria como puerta de entrada al sistema de salud y promover la educación en salud, aclarando las responsabilidades de cada servicio.

Palabras clave: Enfermería; Emergencias; Atención ambulatoria.

\section{Introdução}

O Sistema Único de Saúde (SUS) objetiva, em primeiro lugar, o acesso universal à saúde. Para isso, são colocados em prática os princípios de universalidade, equidade e integralidade, responsabilizando-se pela atenção à saúde da prevenção à recuperação, assim como o atendimento em todos os níveis de atenção e complexidade, acolhendo todo e qualquer cidadão que vive em território brasileiro (Costa, 2017).

A Portaria no 354, de 10 de março de 2014 do Ministério da Saúde define urgência como "ocorrência imprevista de agravo a saúde como ou sem risco potencial a vida, cujo portador necessita de assistência médica imediata", e emergência como "constatação médica de condições de agravo a saúde que impliquem sofrimento intenso ou risco iminente de morte, exigindo, portanto, tratamento médico imediato". Já as situações não urgentes são definidas como aquelas que podem ser encaminhadas a um pronto atendimento ambulatorial, ou para o atendimento ambulatorial convencional (Brasil, 2014; Mena, Piacsek \& Motta, 2017)

Reportando-se à frase "a teoria na prática é outra", pode-se dizer que os serviços de urgência e emergência (UE) são os que mais sofrem com os efeitos da desorganização do sistema de saúde. A falta ou a inadequação das estruturas físicas e tecnológicas de unidades de saúde, a superlotação dos pronto-socorros devido à escassez de solução de problemas corriqueiros da população na Atenção Básica ( $\mathrm{AB})$, o cuidado fragmentado, a não realização da classificação de risco nas portas de entrada das urgências, são algumas das barreiras que prejudicam o serviço de urgência, causando superlotação, insatisfação e problemas no atendimento (Lessa, 2017).

Pesquisas dos Estados Unidos, Canadá, Reino Unido e Austrália relatam que a procura por atendimentos de UE aumenta de 3\% a 6\% a cada ano. No Brasil, essa demanda também aumenta progressivamente. A maior parte dos atendimentos realizados no país é decorrente de problemas menos complexos que poderiam ser resolvidos em serviços de $\mathrm{AB}$ ou especializados ou, ainda, em serviços de urgência de menor complexidade (Coster, Turner, Bradbury \& Cantrell, 2017; Oliveira, 2017). 
Sob esse viés, nos serviços hospitalares de UE a atuação do enfermeiro é essencial, pois envolve particularidades imprescindíveis à gerência do cuidado. Desse modo, o enfermeiro assume papel de destaque, desempenhando atividades assistenciais e gerenciais articuladas, como: previsão e provisão de recursos materiais, liderança da equipe, dimensionamento de pessoal e coordenação de todo o processo assistencial. Além disso, esse profissional deve desenvolver estratégias que fortaleçam o trabalho em equipe e organização da assistência (Sousa, Damasceno, Almeida, Magalhães \& Ferreira, 2019; Santos, Lima, Pestana, Colomé, \& Erdmann, 2016).

De acordo com Will, Farias, Jesus e Rosa (2020), as áreas de Urgência e Emergência requerem do profissional enfermeiro a forma diferenciada do padrão tradicional de modelo Biomédico, organizado por rotinas e protocolos para o guiarem. $\mathrm{O}$ atendimento a ser prestado na emergência é uma assistência que está direcionada aos portadores de quadros agudos, de natureza clínica, traumática ou psiquiátrica. Diante de uma emergência, a Enfermagem tem como dever estabelecer prioridades na assistência em acordo com a avaliação primária da vítima, garantindo a identificação e o tratamento das situações que ameaçam a vida.

Todas as atividades exercidas pelo enfermeiro são regidas pela Lei do Exercício Profissional no 7.498, de 25 de junho de 1986, e pelo Código de Ética dos Profissionais de Enfermagem. Nesse sentido, o atendimento de urgência e emergência abre espaço para muitas discussões éticas e legais no que concerne à atividade profissional e da competência baseada na lei para a realização de procedimentos. Por isso, o momento do atendimento de emergência requer rapidez, destreza, atenção e amparo legal. Dentre as atividades do enfermeiro nos serviços de urgência, destacam-se: punção arterial, punção de jugular externa, uso do Desfibrilador Externo Automático (DEA), prescrição de oximetria contínua e punção intraóssea (Morais Filho, Martini, de Oliveira Vargas, Reibnitz, Bitencourt \& Lazzari, 2016).

Para que prioridades sejam estabelecidas, o enfermeiro conta com uma ferramenta muito importante: a triagem com classificação de risco. Ele é o profissional de referência para realizar essa classificação, sendo respaldado por lei diante à tomada de decisão. A Resolução 661/2021 do Conselho Federal de Enfermagem (COFEN), em seu art. 1º, resolve: "No âmbito da Equipe de Enfermagem, a classificação de Risco e priorização da assistência é privativa do Enfermeiro, observadas as disposições legais da profissão.” (Camargo Neto, Andrade, Karpiuck, \& Ganassin, 2018).

Ademais, a presente pesquisa é relevante visto que os serviços hospitalares de UE são a porta de entrada para todos os hospitais, e, em geral, são os primeiros serviços procurados pela população, desde situações simples até as mais complexas. Além disso, a demanda por esses serviços cresce progressivamente, e esse fato exige um cuidado gerenciado, integral, sistematizado, organizado e satisfatório aos usuários. Consequentemente, o tipo de atendimento em estudo necessita de uma liderança de qualidade, posto que prestará cuidados de enfermagem de maior complexidade (Moreira\& Losekann, 2015; Freire, Araújo, de Brito Araújo, da Silva Alves, Freire, \& de Sousa, 2019).

Ante ao exposto, este estudo buscou respostas ao seguinte questionamento: quais são os tipos de atendimentos ambulatoriais de caráter de urgência realizados no Estado de Minas Gerais e suas implicações no papel da enfermagem? Com o fito de atender a questão proposta, a pesquisa teve como objetivo: Realizar a descrição dos dados disponibilizados pelo Sistema de Informações Ambulatoriais (SIA/SUS) sobre os atendimentos de caráter de urgência.

\section{Metodologia}

Trata-se de um estudo descritivo de abordagem quantitativa. De acordo com Viana e Ferreira (2018), a pesquisa descritiva tem como objetivo primordial a descrição das características de determinada população ou fenômeno ou, então, o estabelecimento de relações entre variáveis. 
Realizou-se uma análise de série temporal dos atendimentos de caráter de urgência através do Sistema de Informações Ambulatoriais (SIA/SUS). Estudos desta natureza possuem celeridade de obtenção dos dados e seleção de informações presentes no sistema (Cassettari \& Mello, 2017).

O estudo foi realizado em três etapas. A primeira foi a coleta de dados secundários, obtidos por meio do SIA/SUS. Os dados gerados para posterior análise limitaram-se à produção ambulatorial do SUS no Estado de Minas Gerais, no período de janeiro a dezembro de 2019. A escolha do período de um ano foi devido à abrangência de informações disponíveis, possibilitando melhor análise e discussão dos dados encontrados.

Posteriormente, na segunda etapa, seguiu-se ao estudo dos resultados encontrados no SIA/SUS. A fim de atingir os objetivos da pesquisa, realizou-se a análise das tabelas considerando o número de atendimentos de caráter de urgência. Ressalta-se que todos os números dos procedimentos supramencionados se enquadram no atendimento desejado.

Para a execução e organização da segunda etapa, optou-se por gerar tabelas dos meses de janeiro a dezembro de 2019, ressaltando os atendimentos de urgência, a fim de comparar os números obtidos. A partir disso, foi criada uma tabela para melhor visualização e comparação dos dados. Essa tabela possui os meses de comparação nas colunas, os grupos de procedimentos nas linhas e o número de atendimento realizado como conteúdo.

Para melhor investigação, foram criadas mais duas tabelas: a primeira abordou a quantidade de vezes que o profissional enfermeiro realizou o atendimento de urgência; a segunda mostrou a quantidade de atendimentos de caráter de urgência em cada nível de complexidade (atenção básica, média complexidade, alta complexidade e "não se aplica").

$\mathrm{Na}$ terceira etapa os dados foram relacionados com diversos artigos científicos que abordavam sobre os atendimentos ambulatoriais de caráter de urgência. Tais estudos possibilitaram a identificação de informações sobre esses atendimentos, bem como os motivos da procura por um serviço hospitalar de urgência e o papel da enfermagem nesses serviços e a sua relevância. Todos os achados contribuíram positivamente para que os objetivos da presente pesquisa fossem alcançados com êxito. Considerando que essa pesquisa foi realizada exclusivamente com bancos de dados, sem possibilidade de identificação individual, sua apreciação por parte do Comitê de Ética em Pesquisa (CEP) é dispensada (Brasil, 2016).

\section{Resultados}

A computação dos dados obtidos no SIA/SUS foi feita a partir da organização de três tabelas. A tabela 1 apresenta a quantidade de atendimentos de caráter de urgência, separados por tipos de procedimentos. 
Tabela 1 - Atendimentos de caráter de urgência em Minas Gerais de janeiro a dezembro de 2019.

\begin{tabular}{|c|c|c|c|c|c|c|c|}
\hline Grupo Procedimento & $\begin{array}{c}\text { Janeiro } \\
2019\end{array}$ & $\begin{array}{l}\text { Fevereiro } \\
2019\end{array}$ & $\begin{array}{c}\text { Março } \\
2019\end{array}$ & $\begin{array}{c}\text { Abril } \\
2019\end{array}$ & $\begin{array}{l}\text { Maio } \\
2019\end{array}$ & $\begin{array}{c}\text { Junho } \\
2019\end{array}$ & TOTAL \\
\hline $\begin{array}{l}\text { Ações de promoçãa e } \\
\text { prevenção em saúde }\end{array}$ & $89 \quad(0,02 \%)$ & $323(0,09 \%)$ & $274(0,06 \%)$ & $190(0,04 \%)$ & $166(0,03 \%)$ & $105(0,02 \%)$ & $1.147(0,04 \%)$ \\
\hline $\begin{array}{l}\text { Procedimentos com } \\
\text { finalidade diagnóstica }\end{array}$ & $73.767(20 \%)$ & $70.403(19,6 \%)$ & $\begin{array}{c}81.342 \\
(18,2 \%)\end{array}$ & $\begin{array}{l}92.493 \\
(20,6 \%)\end{array}$ & $\begin{array}{c}103.722 \\
(21 \%)\end{array}$ & $\begin{array}{c}101.614 \\
(21 \%)\end{array}$ & $523.341(20,1 \%)$ \\
\hline Procedimentos clínicos & $\begin{array}{l}276.742 \\
(74,5 \%)\end{array}$ & $268.288(75 \%)$ & $\begin{array}{c}343.830 \\
(77 \%)\end{array}$ & $\begin{array}{c}337.256 \\
(75 \%)\end{array}$ & $\begin{array}{l}369.886 \\
(74,6 \%)\end{array}$ & $\begin{array}{l}360.913 \\
(74,9 \%)\end{array}$ & $1.956 .915(75,1 \%)$ \\
\hline Procedimentos cirúrgicos & $19.308(5,2 \%)$ & $19.192(5 \%)$ & $\begin{array}{l}20.116 \\
(4,5 \%)\end{array}$ & $19.068(4,2 \%)$ & $21.098(4,2 \%)$ & $18.121(3,8 \%)$ & $116.903(4,5 \%)$ \\
\hline $\begin{array}{l}\text { Transplante de órgãos, } \\
\text { tecidos e células }\end{array}$ & $451 \quad(0,1 \%)$ & $185(0,05 \%)$ & $148(0,03 \%)$ & $143(0,03 \%)$ & $308(0,06 \%)$ & $429(0,09 \%)$ & $1.664(0,06 \%)$ \\
\hline $\begin{array}{l}\text { Órteses, próteses e } \\
\text { materiais especiais }\end{array}$ & $386(0,1 \%)$ & $278(0,07 \%)$ & $251(0,06 \%)$ & $278(0,06 \%)$ & $366(0,07 \%)$ & $400(0,08 \%)$ & $1.959(0,07 \%)$ \\
\hline $\begin{array}{l}\text { Ações complementares de } \\
\text { atenção à saúde }\end{array}$ & $264(0,07 \%)$ & $233(0,06 \%)$ & $832(0,1 \%)$ & $309(0,07 \%)$ & $287(0,05 \%)$ & $312(0,06 \%)$ & $2.237(0,08 \%)$ \\
\hline TOTAL & 371.007 & 358.902 & 446.793 & 449.737 & 495.833 & 481.894 & 2.604 .1666 \\
\hline $\begin{array}{l}\text { Grupo } \\
\text { Procedimento }\end{array}$ & $\begin{array}{c}\text { Julho } \\
2019\end{array}$ & $\begin{array}{l}\text { Agosto } \\
2019\end{array}$ & $\begin{array}{l}\text { Setembro } \\
2019\end{array}$ & $\begin{array}{l}\text { Outubro } \\
2019\end{array}$ & $\begin{array}{l}\text { Novembro } \\
2019\end{array}$ & $\begin{array}{l}\text { Dezembro } \\
2019\end{array}$ & TOTAL \\
\hline $\begin{array}{l}\text { Ações de promoção e } \\
\text { prevenção em saúde }\end{array}$ & $88 \quad(0,02 \%)$ & $850 \quad(0,2 \%)$ & $67 \quad(0,01 \%)$ & $\begin{array}{c}906 \\
(0,16 \%)\end{array}$ & $858 \quad(0,2 \%)$ & $2.283(0,4 \%)$ & $5.025(0,2 \%)$ \\
\hline $\begin{array}{l}\text { Procedimentos com } \\
\text { finalidade diagnóstica }\end{array}$ & $\begin{array}{l}107.383 \\
(24,2 \%)\end{array}$ & $\begin{array}{l}115.725 \\
(21,6 \%)\end{array}$ & $\begin{array}{l}115.142 \\
(23,4 \%)\end{array}$ & $\begin{array}{l}124.093 \\
(24 \%)\end{array}$ & $119.162(26 \%)$ & $\begin{array}{l}143.957 \\
(26,8 \%)\end{array}$ & $725.462(28,1 \%)$ \\
\hline Procedimentos clínicos & $\begin{array}{l}317.245 \\
(71,5 \%)\end{array}$ & $\begin{array}{c}395.543 \\
(74 \%)\end{array}$ & $\begin{array}{c}355.865 \\
(72,32 \%)\end{array}$ & $\begin{array}{l}368.815 \\
(71,5 \%)\end{array}$ & $\begin{array}{l}308.733 \\
(68,5 \%)\end{array}$ & $\begin{array}{l}368.811 \\
(68,6 \%)\end{array}$ & $\begin{array}{c}1.720 .489 \\
(66,7 \%)\end{array}$ \\
\hline Procedimentos cirúrgicos & $17.487(4 \%)$ & $21.345(4 \%)$ & $20.359(4,13 \%)$ & $\begin{array}{l}21.114 \\
(4,1 \%)\end{array}$ & $20.713(5 \%)$ & $21.799(4 \%)$ & $122.817(4,8 \%)$ \\
\hline $\begin{array}{l}\text { Transplante de órgãos, } \\
\text { tecidos e células }\end{array}$ & $367(0,08 \%)$ & $142(0,02 \%)$ & $97 \quad(0,02 \%)$ & $506(0,1 \%)$ & $346(0,08 \%)$ & $315(0,06 \%)$ & $1.773(0,06 \%)$ \\
\hline $\begin{array}{l}\text { Órteses, próteses e } \\
\text { materiais especiais }\end{array}$ & $376(0,08 \%)$ & $313(0,05 \%)$ & $299(0,06 \%)$ & $499(0,1 \%)$ & $396(0,09 \%)$ & $381(0,07 \%)$ & $2.264(0,08 \%)$ \\
\hline $\begin{array}{l}\text { Ações complementares de } \\
\text { atenção à saúde }\end{array}$ & $212(0,04 \%)$ & $301(0,05 \%)$ & $281(0,06 \%)$ & $198(0,04 \%)$ & $328(0,07 \%)$ & $256(0,05 \%)$ & $1.576(0,06 \%)$ \\
\hline TOTAL & 443.158 & 534.219 & 492.110 & 516.131 & 450.536 & 537.802 & 2.579 .406 \\
\hline
\end{tabular}

Fonte: Ministério da Saúde - Sistema de Informação Ambulatorial (SIA/SUS).

A Tabela 1 mostra grande variação na quantidade dos atendimentos de caráter de urgência, destacando-se o mês de dezembro de 2019 com aumento de 87.266 atendimentos em relação a novembro. O mês com menor número de pacientes atendidos e, consequentemente, menor quantidade de procedimentos realizados, foi o de fevereiro. O número médio de atendimentos mensais no ano de 2019 foi de $464.843,5$.

No primeiro semestre de 2019 (janeiro a junho), todos os meses apresentaram crescimento do número de atendimentos, exceto do mês de janeiro para fevereiro e maio para junho, com diminuição de 12.105 e 13.939 pessoas acolhidas na urgência, respectivamente. Além disso, a maior diferença obtida foi do mês de fevereiro para março, com um crescimento de 87.891 procedimentos urgentes realizados.

No segundo semestre (julho a dezembro) a quantidade de procedimentos sofreu maiores variações do que no primeiro semestre. Os meses de julho, setembro e novembro foram os que obtiveram menor número de atendimentos, destacando-se a diferença de outubro para novembro, com queda de 65.595 pacientes. 
A Tabela 2, apresentada a seguir, refere-se à quantidade de atendimentos de caráter de urgência realizados por profissionais enfermeiros.

Tabela 2 - Quantidade de atendimentos de caráter de urgência por profissionais enfermeiros em Minas Gerais de janeiro a dezembro de 2019.

\begin{tabular}{|c|c|c|c|c|c|c|c|}
\hline Meses & Janeiro & Fevereiro & Março & Abril & Maio & Junho & TOTAL \\
\hline & 2019 & 2019 & 2019 & 2019 & 2019 & 2019 & \\
\hline $\begin{array}{l}\text { Quantidade de } \\
\text { atendimentos }\end{array}$ & $\begin{array}{c}124.224 \\
(14 \%)\end{array}$ & $117.914(13 \%)$ & $154.657(18 \%)$ & $\begin{array}{c}147.965 \\
(17 \%)\end{array}$ & $164.996(19 \%)$ & $162.992(19 \%)$ & 872.748 \\
\hline \multirow[t]{2}{*}{ Meses } & Julho & Agosto & Setembro & Outubro & Novembro & Dezembro & TOTAL \\
\hline & 2019 & 2019 & 2019 & 2019 & 2019 & 2019 & \\
\hline $\begin{array}{l}\text { Quantidade de } \\
\text { atendimentos }\end{array}$ & $\begin{array}{c}148.263 \\
(14 \%)\end{array}$ & $\begin{array}{c}192.143 \\
(19 \%)\end{array}$ & $171.000(17 \%)$ & $\begin{array}{c}174.421 \\
(17 \%)\end{array}$ & $150.666(15 \%)$ & $182.212(18 \%)$ & 1.018 .705 \\
\hline
\end{tabular}

Fonte: Ministério da Saúde - Sistema de Informação Ambulatorial (SIA/SUS).

O mês de agosto apresentou maior número de atendimentos realizados pela enfermagem. No primeiro semestre de 2019 (janeiro a junho) houve pequena variação na quantidade de atendimentos por profissionais enfermeiros entre os meses, exceto entre fevereiro e março, com um salto de 36.743 atendimentos, e entre abril e maio, com um salto de 17.031 atendimentos. A média de atendimentos do semestre foi de 145.458.

No segundo semestre de 2019 (julho a dezembro) a variação do número de atendimentos realizados por profissionais enfermeiros foi maior. Entre julho e agosto observa-se um aumento de 43.880 atendimentos. Já no mês de setembro, o número cai, passando de 192.143 para 171.000 (diferença de 21.143 atendimentos). Entre novembro e dezembro é possível perceber outro grande aumento, de 31.546 atendimentos. A média de atendimentos do semestre foi de 169.784,16.

A seguir, a tabela 3 demonstra a quantidade de atendimentos de caráter de urgência por nível de complexidade. 
Tabela 3 - Quantidade de atendimentos de caráter de urgência por nível de complexidade em Minas Gerais de janeiro a dezembro de 2019.

\begin{tabular}{|c|c|c|c|c|c|c|}
\hline Complexidade & $\begin{array}{c}\text { Janeiro } \\
2019\end{array}$ & $\begin{array}{c}\text { Fevereiro } \\
2019\end{array}$ & $\begin{array}{c}\text { Março } \\
2019\end{array}$ & $\begin{array}{c}\text { Abril } \\
2019\end{array}$ & $\begin{array}{l}\text { Maio } \\
2020\end{array}$ & $\begin{array}{c}\text { Junho } \\
\mathbf{2 0 2 0}\end{array}$ \\
\hline Atenção Básica & $\begin{array}{l}7.435 \\
(2 \%)\end{array}$ & $6.115(1,7 \%)$ & $7.598(1,7 \%)$ & $6.081(1,3 \%)$ & $7.591(1,5 \%)$ & $5.729(1,2 \%)$ \\
\hline Média Complexidade & $\begin{array}{l}349.664 \\
(94,2 \%)\end{array}$ & $341.037(95 \%)$ & $\begin{array}{l}426.484 \\
(95,5 \%)\end{array}$ & $\begin{array}{c}431.150 \\
(96 \%)\end{array}$ & $\begin{array}{l}474.666 \\
(95,8 \%)\end{array}$ & $462.956(96 \%)$ \\
\hline Alta Complexidade & $\begin{array}{l}13.258 \\
(3,6 \%)\end{array}$ & $11.239(3,2 \%)$ & $11.628(2,6 \%)$ & $\begin{array}{l}11.919 \\
(2,6 \%)\end{array}$ & $12.923(2,6 \%)$ & $12.497(2,7 \%)$ \\
\hline Não se aplica & $\begin{array}{c}650 \\
(0,2 \%)\end{array}$ & $511(0,1 \%)$ & $1.083(0,2 \%)$ & $587 \quad(0,1 \%)$ & $653(0,1 \%)$ & $712(0,1 \%)$ \\
\hline TOTAL & 371.007 & 358.902 & 446.793 & 449.737 & 495.833 & 481.894 \\
\hline Complexidade & $\begin{array}{c}\text { Julho } \\
2019\end{array}$ & $\begin{array}{c}\text { Agosto } \\
2019\end{array}$ & $\begin{array}{c}\text { Setembro } \\
2019\end{array}$ & $\begin{array}{c}\text { Outubro } \\
2019\end{array}$ & $\begin{array}{c}\text { Novembro } \\
2019\end{array}$ & $\begin{array}{c}\text { Dezembro } \\
2019\end{array}$ \\
\hline Atenção Básica & $\begin{array}{c}8.338 \\
(1,9 \%)\end{array}$ & $9.479(1,8 \%)$ & $8.265(1,7 \%)$ & $9.297(1,8 \%)$ & $8.238(1,8 \%)$ & $17.100(3,2 \%)$ \\
\hline Média Complexidade & $\begin{array}{c}420.801 \\
(95 \%)\end{array}$ & $\begin{array}{l}510.245 \\
(95,5 \%)\end{array}$ & $\begin{array}{l}469.139 \\
(95,3 \%)\end{array}$ & $\begin{array}{l}493.401 \\
(95,6 \%)\end{array}$ & $428.377(95 \%)$ & $\begin{array}{l}506.644 \\
(94,2 \%)\end{array}$ \\
\hline Alta Complexidade & $\begin{array}{c}13.431 \\
(3 \%)\end{array}$ & $13.881(2,6 \%)$ & $14.126(2,9 \%)$ & $\begin{array}{l}12.736 \\
(2,5 \%)\end{array}$ & $13.197(3 \%)$ & $13.421(2,5 \%)$ \\
\hline Não se aplica & $\begin{array}{c}588 \\
(0,1 \%)\end{array}$ & $614(0,1 \%)$ & $580(0,1 \%)$ & $697 \quad(0,1 \%)$ & $724 \quad(0,2 \%)$ & $637 \quad(0,1 \%)$ \\
\hline TOTAL & 443.158 & 534.219 & 492.110 & 516.131 & 450.536 & 537.802 \\
\hline
\end{tabular}

Fonte: Ministério da Saúde - Sistema de Informação Ambulatorial (SIA/SUS).

Tratando-se da $\mathrm{AB}$, é possível observar um grande aumento na quantidade de atendimentos no mês de dezembro, com aumento de $107,57 \%$ em relação a novembro. A média anual de atendimentos da AB foi de 8.438,83.

A quantidade de atendimentos da Média Complexidade (MC) merece destaque, pois se apresenta muito maior do que os outros níveis. Durante o ano percebe-se uma pequena variação na quantidade de atendimentos da MC, exceto os meses de agosto e dezembro, que representam os meses com maiores números, 510.245 e 506.644 respectivamente. A média anual de atendimentos da MC foi de 442.880,33.

No que concerne à Alta Complexidade (AC), o mês de setembro chama atenção, pois representa o mês com maior número de atendimentos. A variação da quantidade de atendimentos da AC durante o ano é pequena. A média anual de atendimentos da AC foi de 12.854,6.

\section{Discussão}

A análise dos dados evidenciou escassez de números no que tange a ações de promoção e prevenção à saúde, ações que, na maioria das vezes, poderiam diminuir ou até mesmo evitar a procura pelos serviços de urgência. A promoção da saúde incentiva o indivíduo a desenvolver habilidades que promovam sua adaptação às fases do seu ciclo de vida e aos processos de saúde e doença de forma satisfatória. Já a prevenção de complicações está relacionada aos problemas iminentes do paciente e aos fatores de risco inerentes ou exteriores ao indivíduo (Freire et al. 2016).

A presença do enfermeiro nos serviços de urgência é obrigatória, com o objetivo de atender os pacientes críticos, respeitando as atribuições e competências pertencentes a esse profissional (da Cruz, Wanzeler, de Sena Bastos, Pinheiro, dos Santos, Fayal, \& Barbosa, 2020). Uma das competências exclusivas do enfermeiro na urgência é a triagem, respaldada pelo Conselho Regional de Enfermagem (COREN) e conceituada como o primeiro contato com o paciente. É nesse atendimento 
que o profissional faz a avaliação e a classificação de risco do usuário, permitindo que os mais graves tenham prioridade em relação aos demais, entretanto sem negar atendimento aos casos simples (Silva \& Invenção, 2018).

A grande quantidade de atendimentos realizados pelos profissionais enfermeiros pode ser justificada pelo déficit de profissionais e pela alta demanda de pacientes que poderiam ser atendidos na rede básica de saúde (Silva \& Invenção, 2018). Segundo Cruz et al. (2020), o déficit de profissionais não se restringe à equipe de enfermagem, mas abrange os profissionais da medicina, posto que algumas condutas assumidas pelo enfermeiro que são de responsabilidade médica. Muitos médicos se ausentam na liderança da prática clínica do diagnóstico e prescrição médica, prejudicando o processo de enfermagem e sobrecarregando o serviço.

O serviço de urgência recebe diversos casos, desde o mais simples, que poderiam ser resolvidos na $\mathrm{AB}$, até os mais complexos, resolvidos nos serviços de média e alta complexidade. A crescente procura pelo atendimento ambulatorial nos prontos socorros representa que os usuários só procuram o serviço porque não tiveram acesso a nenhum outro local. A falta de organização e de comunicação entre os níveis de atenção gera desordem no sistema e coloca a vida dos pacientes em risco (Camargo Neto, Andrade, Karpiuck, \& Ganassin, 2018).

Pereira, de Oliveira Flausino, Barbosa, dos Santos Ferreira, Correia, e Lima (2019) avaliando o perfil da demanda dos clientes que procuram atendimento no serviço de UE, afirma que a maioria dos pacientes atendidos em emergências clínicas foram adultos jovens, gênero feminino, cor branca, casados e ativos no trabalho. As causas do atendimento mais prevalentes eram desconhecidas e as especialidades clínicas foram, respectivamente, neurologia, cardiologia e pneumologia.

Os diagnósticos para os pacientes que procuram o serviço de UE normalmente foram dor precordial, cefaléia e acidente vascular encefálico. Tratando-se da rota final dos pacientes, na emergência clínica, o maior número foi de alta médica da unidade de pronto atendimento da emergência, se diferenciando pouco entre os que internaram e em seguida receberam alta hospitalar, sendo que 11,5\% morreram (Pereiraet al., 2019).

Segundo uma pesquisa realizada por Strada, Brave, Valpiani, Bentivegna, e Carradori (2019), a alta demanda por serviços de UE tem como consequência a superlotação desses setores. O estudo relata que a manifestação de novas necessidades de saúde, o envelhecimento da população, o extenso número de pacientes com casos complexos e o advento de novas tecnologias de diagnóstico podem contribuir para a crescente procura por serviços hospitalares.

Oliveira, Santos e Roquete (2017) apresentam que o perfil de saúde da população, de prevalência das doenças crônicas e causas externas (CE), causa impacto no padrão de utilização dos serviços de saúde. Isso justifica o maior número de atendimentos nos serviços de maior complexidade, e um menor número de atendimentos realizados na $\mathrm{AB}$.

Na maior parte dos países, a população tem acesso contínuo aos estabelecimentos de urgência e emergência, apesar da atenção primária estar disponível. Reforça-se a grande necessidade da garantia do acesso à atenção primária para os usuários, e consequentemente, um "alívio" para os serviços de urgência e emergência. A maioria dos acometimentos das vítimas que procuram o serviço de urgência envolve pacientes em condições não urgentes (Raknes \& Hunskaar, 2017).

A consequência do grande aumento da demanda pelo atendimento de urgência e emergência engloba quesitos como aglomeração no serviço, aumento dos custos, longo tempo de espera e sobrecarga do serviço. Estudos revelam que esse aumento se deve ao crescimento da população idosa, que muitas vezes possui necessidade de atendimentos mais complexos; aos pacientes que ignoram as orientações recebidas na atenção básica e vão diretamente ao serviço de urgência; populações que envelhecem e adquirem condições crônicas e como consequência possuem necessidades mais complexas; entre outros fatores (Costeret al., 2017).

Para melhor organização do trabalho e efetividade clínica, existem dispositivos da Política Nacional de Humanização (PNH), e os serviços de UE não poderiam estar de fora. A exemplo tem-se o Acolhimento com Classificação de Risco (ACR), que promove humanização no atendimento e trabalho da equipe multidisciplinar, possibilitando mais agilidade no atendimento 
por meio da realização de instrumentos de avaliação prévia. Isso permite que o profissional selecione com segurança as prioridades de atendimento conforme o nível de complexidade clínica e as necessidades do paciente (Sousaet al., 2019).

Dentro dos serviços de urgência e emergência os pacientes passam pela triagem, local no qual o enfermeiro possui competência para atuar. Esse é um profissional que entende as necessidades do paciente; aplica seu conhecimento e desenvolve relacionamento com os pacientes e seus familiares; presta cuidado integral, com assistência centrada no paciente, focada na prática ética, nas relações e na gerência profissional; pode utilizar métodos avançados de melhoria da qualidade do serviço (Usui \&Yamauchi, 2019).

Os enfermeiros possuem papel relevante nas equipes de saúde, garantindo atendimento de alta qualidade. São responsáveis pelo atendimento contínuo ao paciente durante as 24 horas do dia, com atividades que variam de cuidados de enfermagem propícios a acompanhamento de paciente durante procedimentos médicos. Além disso, ainda são responsáveis pela coordenação e supervisão do serviço, contendo serviços de planejamento, provimento de suprimentos, gerenciamento e liderança de equipes, coordenando a prestação de cuidados e executando procedimentos de enfermagem apropriados (Santana, 2019).

Os pacientes, reiteradamente, buscam o serviço terciário como porta de entrada mais rápida, por não terem seus problemas de saúde resolvidos no nível primário de atenção. Essa circunstância faz com que haja prejuízos de cobertura, acessibilidade e resolutividade no cuidado ofertado. Os usuários, apesar de admitirem a importância da AB no sistema de saúde, não a colocam como serviço preferencial como porta de entrada, preferindo procurar níveis de maior complexidade por causa de problemas de saúde de menor complexidade. À vista disso, destaca-se a importância da inserção da educação em saúde no cotidiano dos profissionais, tarefa que o enfermeiro é capacitado para cumprir (de Brito, Albuquerque, Ribeiro, Ponte, Moreira, \& Linhares, 2018).

A AB está sendo substituída pelos ambulatórios e serviços de média e alta complexidade. Muitos usuários acreditam que há maior resolutividade dos seus problemas de saúde na atenção médico-hospitalar, provocando aumento da demanda nos serviços de média complexidade e nas urgências e emergências. Para modificar essa situação a capacitação dos profissionais e a conscientização dos usuários são de grande importância. Além disso, a melhoria da qualidade e agilidade dos atendimentos e a organização do fluxo dos mesmos poderiam colocar um fim na superlotação hospitalar (Siochetta, da Silva, Beuren, \& de Almeida, 2019).

\section{Conclusão}

Os serviços de urgência recebem casos simples e complexos, gerando alta demanda pela população e, concomitantemente, a superlotação desses setores. Por isso, a presente pesquisa reforçou a necessidade da valorização dos profissionais de enfermagem e do fortalecimento da $\mathrm{AB}$ como porta de entrada do sistema de saúde. Outrossim, promover a educação em saúde é importante para esclarecer aos usuários as atribuições de cada serviço, a fim de que não ocorra a sobrecarga de nenhum. Estudos futuros devem ser realizados, visto que há ausência de pesquisas sobre o tema abordado.

\section{Referências}

Resolução n. 510, de 07 de abril de 2016. Denominando-a normas aplicáveis a pesquisas em Ciências Humanas e Sociais. https://bvsms.saude.gov.br.

Resolução n. 661, de 11 de março de 2021. Atualiza e normatiza, no âmbito do Sistema Cofen/Conselhos Regionais de Enfermagem, a participação da Equipe de Enfermagem na atividade de Classificação de Risco. http://www.cofen.gov.br/resolucao-cofen-no-661-2021_85839.html.

de Brito, G. V., Albuquerque, I. M. A. N., Ribeiro, M. A., Ponte, E. C. S., Moreira, R. M. M., \& Linhares, M. D. G. C. (2018). Consulta de Puericultura na Estratégia Saúde da Família: Percepção de Enfermeiros. Revista de APS, 21(1).

Camargo Neto, O., Andrade, G. K. S. D., Karpiuck, L. B., \& Ganassin, A. R. (2018). A Atuação do Enfermeiro no Sistema de Acolhimento e Classificação de Risco nos Serviços de Saúde. J. health sci (Londrina). 
Cassettari, S. D. S. R., \& Mello, A. L. S. F. D. (2017). Demanda e tipo de atendimento realizado em Unidades de Pronto Atendimento do município de FLORIANÓPOLIS, Brasil. Texto \& Contexto-Enfermagem, 26(1).

Costa, D. R. (2017). Auditoria de contas ambulatoriais no sistema único de saúde no estado do Piauí. Revista de Administração em Saúde, 17(68).

Coster, J. E., Turner, J. K., Bradbury, D., \& Cantrell, A. (2017). Why do people choose emergency and urgent care services? A rapid review utilizing a systematic literature search and narrative synthesis. Academic emergency medicine, 24(9), 1137-1149.

da Cruz, A. B., Wanzeler, K. M., de Sena Bastos, D. A., Pinheiro, P. D. N. Q., dos Santos, É. A. F., Fayal, Y. L., \& Barbosa, E. V. (2020). Processo de enfermagem em práticas de urgência e emergência: relato de experiência. Revista Eletrônica Acervo Saúde, (38), e1857-e1857.

Freire, G. V., Araújo, E. T. H., de Brito Araújo, E., da Silva Alves, L., Freire, A. C. M., \& de Sousa, G. F. (2019). Liderança do enfermeiro nos serviços de urgencia e emergencia: revisão integrativa/Nursing leadership in urgency and emergency services: integrative review. Brazilian Journal of Health Review, 2(3), 2029-2041.

de Albuquerque Freire, R. M., Landeiro, M. J. L., Martins, T., \& Peres, H. H. C. (2016). Um olhar sobre a promoção da saúde e a prevenção de complicações: diferenças de contextos. Revista Latino-Americana de Enfermagem, 24, e2749.

Lessa, G. (2017). Análise da política de atenção as urgências em Santa Catarina.

da Motta, M. V., Mena, H., \& Piacsek, G. (2017). Urgência e Emergência. Os conceitos frente às normas administrativas e legais e suas implicações na clínica médica. Saúde, Ética \& Justiça, 22(2), 81-94.

Morais Filho, L. A., Martini, J. G., de Oliveira Vargas, M. A., Reibnitz, K. S., Bitencourt, J. V. O. V., Lazzari, D. (2016). Competência legal do enfermeiro na urgência/emergência. Rev. Enfermagem em Foco, 7 (1): 18-23.

Moreira, A. D. (2015). Principais causas da superlotação de uma emergência em um Hospital privado de Porto Alegre.

Oliveira, F. E. G., Santos, A. S., \& Roquete, F. F. (2017). O uso das informações de custo em serviços públicos de urgência e emergência. Rev. Gestão \& Saúde (Brasília) Vol, 8, 230-253.

Pereira, J. A., Ruas, J. P. P., de Oliveira Flausino, V., Barbosa, A. F. N., dos Santos Ferreira, T. V., Correia, T. C., \& Lima, B. A. L. (2019). Perfil epidemiológico da demanda em unidades de emergência hospitalar: uma revisão de literatura. Revista Eletrônica Acervo Saúde, (32), e1178-e1178.

Raknes, G., \& Hunskaar, S. (2017). Reasons for encounter by different levels of urgency in out-of-hours emergency primary health care in Norway: a cross sectional study. BMC emergency medicine, 17(1), 19.

Santana, R. D. S., Fontes, F. L. D. L., Morais, M. J. D. A., Costa, G. D. S., Silva, R. K. D., Araújo, C. S. D., \& Pereira, R. I. D. N. (2019). Estresse ocupacional dos enfermeiros de urgência e emergência de um hospital público de Teresina (PI). Revista Brasileira de Medicina do Trabalho, 17 (1), 76-82.

Santos, J. L. G. D., Lima, M. A. D. D. S., Pestana, A. L., Colomé, I. C. D. S., \& Erdmann, A. L. (2016). Estratégias utilizadas pelos enfermeiros para promover o trabalho em equipe em um serviço de emergência. Revista Gaúcha de Enfermagem, 37(1).

Silva, A. M. S. M., \& Invenção, A. S. (2018). A atuação do enfermeiro no atendimento de urgência e emergência. UNILUS Ensino e Pesquisa, $15(39), 5-13$.

Sousa, K. H. J. F., Damasceno, C. K. C. S., Almeida, C. A. P. L., Magalhães, J. M., \& Ferreira, M. D. A. (2019). Humanization in urgent and emergency services: Contributions to nursing care. Rev. Gauch. Enferm, 40, e20180263.

Strada, A., Bravi, F., Valpiani, G., Bentivegna, R., \& Carradori, T. (2019). Do health care professionals' perceptions help to measure the degree of overcrowding in the emergency department? A pilot study in an Italian University hospital. BMC Emergency Medicine, $19(1), 47$.

Siochetta, T. M., da Silva, A., Beuren, A. C., \& de Almeida, S. D. M. (2019). Baixa resolutividade na rede de atenção à saúde: um problema vigente. Revista saúde integrada, 12(23), 190-203.

Usui, M. \& Yamauchi, T. (2019). Orientando pacientes para o cuidado adequado: desenvolvendo as competências do enfermeiro de triagem ambulatorial japonês. Nagoya Journal of Medical Science, 81 (4), 597.

Viana, L. S., \& de Andrade Ferreira, M. D. F. (2018). A Educação Ambiental na formação inicial do professor-pedagogo: um estudo descritivo no sudoeste da Bahia. Revista Brasileira de Educação Ambiental (RevBEA), 13(2), 308-331.

Will, R. C., Farias, R. G., Jesus, H. P. D., \& Rosa, T. (2020). Cuidados de enfermagem aos pacientes politraumatizados atendidos na emergência. Nursing (Säo Paulo), 3766-3777. 